In all three groups treatment relieved symptoms quickly and lowered plasma glucose concentrations. In addition the $\mathrm{Hb} \mathrm{\textrm {A } _ { 1 }}$ concentrations fell in all patients, but even at four months after diagnosis the values remained above the normal range for nondiabetics. Previous studies ${ }^{8} 9$ have suggested that the $\mathrm{Hb} \mathrm{A_{1 }}$ concentration probably reflects the mean blood glucose concentration over the previous one to two months, and the results of our study confirm this view. The rate of fall of the $\mathrm{Hb} \mathrm{A}_{1}$ concentration with treatment was uniform in all three groups, which is compatible with the fact that glycosylation of haemoglobin is an irreversible process. ${ }^{10}$ Consequently the $\mathrm{Hb} \mathrm{A}_{1}$ concentration will fall only as new red cells that are glycosylated at a lower prevailing blood glucose concentration are produced, so diluting the higher $\mathrm{Hb} \mathrm{A}_{1}$ concentrations in older red cells.

Measuring the $\mathrm{Hb} \mathrm{A}_{1}$ concentration at diagnosis in maturityonset diabetics may be a useful additional means of predicting those patients likely to respond to diet alone and those likely to require treatment with an oral hypoglycaemic agent. It also reliably assesses diabetic control when new methods of treating diabetics are to be compared, replacing the traditional but less satisfactory estimations of urinary and plasma glucose concentrations.

We are grateful to Pfizer Ltd for support in setting up the assay for glycosylated haemoglobins.

\author{
References \\ ${ }^{1}$ Bunn, H F, et al, Biochemistry and Biophysics Research Communications, \\ $1975,67,103$. \\ ${ }^{2}$ Fluckiger, R, et al, Diabetologia, 1977, 13, 393. \\ ${ }^{3}$ Koenig, R J, et al, New England fournal of Medicine, 1976, 295, 417. \\ 4 Ditzel, J, and Kjaergaard, J-J, British Medical fournal, 1978, 1, 741. \\ ${ }^{5}$ Kynoch, P A M, and Lehmann, H, Lancet, 1977, 2, 16. \\ ${ }^{6}$ Clarke, B F, and Campbell, I W, Lancet, 1975, 1, 246. \\ 7 Paulsen, E P, and Koury, M, Diabetes, 1976, 25, suppl No 2, p 890. \\ ${ }^{8}$ Gonen, B, et al, Lancet, 1977, 2, 734. \\ ${ }^{9}$ Gabbay, K H, et al, fournal of Clinical Endocrinology and Metabolism, 1977, \\ 44, 859. \\ ${ }^{10}$ Peterson, C M, and Jones, R L, Annals of Internal Medicine, 1977, 87, 489.
}

(Accepted 16 February 1979)

\title{
Precision in estimating gestational age and its influence on sensitivity of alphafetoprotein screening*
}

\author{
C J ROBERTS, B M HIBBARD, D R EVANS, K T EVANS, K M LAURENCE, \\ MARGUERITA HOOLE, E ROBERTS, W P ENNIS
}

British Medical fournal, 1979, 1, 981-983

\section{Summary and conclusions}

The interpretation of maternal serum $\alpha$-fetoprotein (AFP) concentrations in relation to fetal neural tube defects depends on accurate assessment of the gestational age. In a quadruple-blind study three antenatal methods of assessment-namely, menstrual dates, clinical examination, and ultrasound scanning-were correlated with postnatal assessment using the Dubowitz scoring system. The best agreement to \pm 1 week was obtained using menstrual dates and ultrasound in combination, such agreement being found in $91(77 \%)$ of the 118 women studied.

Since serum AFP concentrations vary with gestational age, precise gestational dating is necessary. In many cases, particularly in women who are unsure of their dates or have irregular menstrual cycles, ultrasound examination is needed to supplement clinical findings.

*This study was conducted as part of the South Wales Anencephaly and Spina Bifida Study.

Welsh National School of Medicine, Cardiff CF4 4XN C J ROBERTS, MD, PHD, professor of community medicine B M HIBBARD, MD, FRCOG, professor of obstetrics and gynaecology D R EVANS, MB, MRCP, lecturer in child health

K T EVANS, FRCP, FRCR, professor of diagnostic radiology

K M LAURENCE, DSC, FRCPATH, professor of paediatric research

MARGUERITA HOOLE, SRN, SCM, research midwife, South Wales

Anencephaly and Spina Bifida Survey Group

E ROBERTS, DRC, instructor in diagnostic techniques

W P ENNIS, analyst programmer, department of community medicine

\section{Introduction}

The report of the UK Collaborative Study on using serum $\alpha$-fetoprotein (AF) estimations to detect neural tube malformations ${ }^{1}$ pointed out that the screening test for maternal serum AFP concentrations may be affected by the accuracy with which gestational age is measured. Because only one centre in the study reported more than $2 \%$ of pregnancies with doubtful gestational ages they were unable to examine this problem. Yet in the 1970 survey of British births gestational age was uncertain in $17 \%$ of cases, ${ }^{2}$ and Beazley and Underhill ${ }^{3}$ found uncertain dates in $22 \%$ of patients. The early experience of our group suggested a similar figure. Unlike in most biochemical investigations the transition of AFP concentrations from normal to abnormal values is related to time, and the accuracy of estimations of gestational age is emerging as a key issue in the interpretation of AFP concentrations.

There are three principal methods of assessing gestational age in mid-pregnancy - namely, from the date of the last menstrual period; from clinical examination of the size of the uterus; and from an ultrasound examination. The final estimate may be a composite of all these. In addition, gestational age may be assessed shortly after birth by the Dubowitz scoring system. ${ }^{4}$ All these methods inevitably suffer from some degree of observer variation and are subject to normal biological variation. We have carried out an investigation to study the accuracy with which gestational age may be estimated in mid-pregnancy and the possible influence that any imprecision might have on the accuracy of subsequent classification of the patient's AFP concentration as normal or abnormal. We used the Dubowitz assessment as the independent standard against which the other methods were judged.

\section{Patients and methods}

We initially admitted into the study 131 women attending for booking at the professorial antenatal clinics at the University Hospital 
of Wales but subsequently excluded 13 because of errors in data recording (eight), twins (four), and neonatal death (one). Each patient underwent three independent assessments of gestational age on the same day, each being done blind of the others. A research midwife (MH) supervised the whole procedure to ensure that the blind strategy was adhered to properly. The three methods of estimation were as follows.

Last menstrual period-The gestational age was calculated from the stated date of the last menstrual period and was rounded to the nearest week, one to three days over a complete week being rounded down and four days and more being rounded up. In addition details of the length and regularity of the menstrual cycle and previous use of oral contraception were recorded.

Clinical examination-Each patient emptied her bladder before being examined. The uterine size was determined by both abdominal palpation and bimanual examination.

Ultrasound examination-The biparietal diameter was measured on a static B scan display, with one operator (ER) interpreting all the measurements. The fetal age was deduced from the biparietal diameter, using the standards of Campbell and Newman. ${ }^{5}$

The Dubowitz scores were all assessed by one observer (DE) within 72 hours after birth, again without knowledge of the previous estimations of gestational age, and were used as the independent standard.

\section{Results}

Gestational age estimated from menstrual data alone agreed to within \pm 1 week with that calculated from the Dubowitz score in 80 cases $(67.8 \%)$, and that estimated from ultrasound alone agreed in 81 cases $(68.6 \%$ ) (table I). Ages estimated from clinical examination correlated in only 71 cases $(60 \cdot 2 \%)$. The degree of agreement between the method using the date of the last menstrual period and the Dubowitz score was achieved even though 44 patients $(37 \%)$ reported irregular menstrual cycles or were uncertain of their dates, or both. In the 74 mothers who had regular cycles and were certain of their dates concordance increased to $77 \%$ (57 patients; table II). By combining the results obtained in the women with reliable menstrual dates with those obtained from supplementary ultrasound examination in the remainder concordance was achieved in 91 cases $(77 \cdot 1 \%)$. Overestimation of gestational age was most common when the date of the last menstrual period was used (by two weeks or more in 31 patients $(26.3 \%)$ ), and underestimation was commonest with ultrasound (two weeks or more in 25 patients $(21 \cdot 2 \%)$ ).

\section{Discussion}

The purpose of this study was to examine, under field conditions, the accuracy of each of the three usual methods of estimating gestational age antenatally, taking due account of the substantial proportion of women who are uncertain of the date of their last menstrual period. To do this we had to select a fourth measurement against which each of these three methods could be compared and which, as far as this study was concerned, was independent of the antenatal findings. We chose the Dubowitz assessment for this purpose. Originally this standard was constructed from estimates of gestational age calculated from the known date of the last menstrual period but it has long since become established in its own right. The results given in the tables are in no way confounded by the original interdependence of the Dubowitz examination on menstrual data.

The present investigation was unusual in that four methods of judging gestational age were used simultaneously on the same cohort, with each estimator being totally unaware of the results obtained by his colleagues. Although for the whole sample agreement between the date of the last menstrual period and the Dubowitz score was virtually as good as that between ultrasound and the score, in a substantial number of women the last menstrual period could not be used because the dates were uncertain or the cycle irregular. Thirty per cent of the patients enrolled fell into this category. In addition, 12 women were initially excluded because they could not give even an approximate date for their last menstrual period
TABLE I-Agreement of estimates of gestational age obtained with three different methods with results of Dubowitz examination in 118 births. (Figures are numbers (\%) of births)

\begin{tabular}{|c|c|c|c|c|c|}
\hline & \multicolumn{2}{|c|}{ Underestimate } & \multirow{2}{*}{$\begin{array}{c}\text { Agreement } \\
\text { to within } \\
\pm 1 \text { week }\end{array}$} & \multicolumn{2}{|c|}{ Overestimate } \\
\hline & $\geqslant 3$ weeks & 2 weeks & & 2 weeks & $\geqslant 3$ weeks \\
\hline $\begin{array}{l}\text { Date of last menstrual perioc } \\
\text { Clinical examination } \ldots \\
\text { Ultrasound } \quad .\end{array}$ & $\begin{array}{l}4(3 \cdot 4) \\
6(5 \cdot 1) \\
8(6 \cdot 8)\end{array}$ & $\begin{array}{l}3(2 \cdot 5) \\
16(13 \cdot 5) \\
17(14 \cdot 4)\end{array}$ & $\begin{array}{l}80(67 \cdot 8) \\
71(60 \cdot 2) \\
81(68 \cdot 6)\end{array}$ & $\begin{array}{l}11(9 \cdot 3) \\
15(12 \cdot 7) \\
8(6 \cdot 8)\end{array}$ & $\begin{array}{r}20(17 \cdot 0) \\
10(8 \cdot 5) \\
4(3 \cdot 4)\end{array}$ \\
\hline
\end{tabular}

TABLE II-Effect of using combinations of methods to estimate gestational age on agreement of results with Dubowitz score

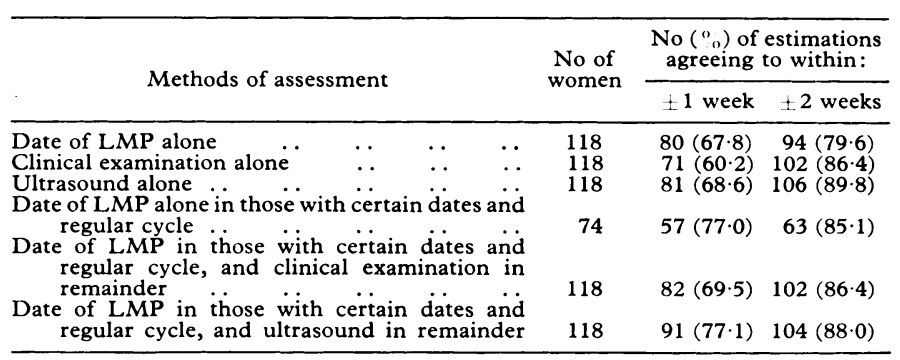

LMP = Last menstrual period.

The sensitivity of pregnancy dating required for interpreting serum AFP concentrations is much greater than has hitherto been needed in clinical practice. In the present study clinical examination was the poorest correlate at \pm 1 week but was nearly as accurate as ultrasound at \pm 2 weeks (table II). Thus gross deviations in size are likely to be detected by clinical examination and alert the obstetrician to major errors in dates, the possibility of multiple pregnancy, missed abortion, or other uterine and pelvic abnormality.

In our population about $10 \%$ of those screened had serum AFP concentrations lying within the "critical range"-that is, the range in which the decision to offer, or not to offer, amniocentesis could be reversed by an alteration of as little as one week in the estimate of gestational age. Some idea of the frequency with which original estimates of gestational age are subsequently altered may be seen from our own investigation and from the study by Ferguson-Smith et al. ${ }^{6}$ In their study $35 \%$ of subjects who were "serum-positive" at the first test and subsequently classified as "serum-negative" at the second test were considered to have been misclassified at the initial test because the gestational age had been underestimated. In our study, over three months in one clinic, 25 out of $30(83 \%)$ serum AFP estimations that had been classified initially as abnormal were later reclassified as normal after an upward revision of the gestational age. In half the cases the upward revision was three weeks or more. An initial underestimate of gestational age would have the effect of resetting the cut-off point for amniocentesis at an appreciably lower level and to some degree would act as a safety device.

It is not clear in how many cases the gestational age should be revised downward, bringing the AFP concentrations into the range requiring further investigation. ${ }^{7}$ Without such revisions many false-negative results are inevitable. Yet in the present study there was a greater tendency to overestimate rather than underestimate gestation when menstrual dates alone were used (as might be expected from clinical factors such as amenorrhoea occurring after oral contraception has been discontinued).

Procedures that use two serum AFP estimates separated by a month are claimed by some ${ }^{16}$ to be more accurate than those that rely on a single estimate. This, however, may simply reflect the opportunity conferred by the time interval to make a more accurate estimation of gestational age. The narrower range of gestational age recommended for screening in the UK Collaborative Study is based principally on the belief that individual output of AFP is least variable in the recommended age range, but it might reflect that estimations of gestational age are more reliable at this period. 
The effect of inaccuracies in assessing gestational age alone in terms of final management may be estimated. With $60 \%$ concordance nearly $2 \%$ of all women may be subjected to amniocentesis when they do not really need it while in $2 \%$ the test may be omitted when it is actually required. Even with $90 \%$ concordance (unattainable in practice at present) the false-positive and false-negative rates are still over $1 \%$. Expressing this differently, with an amniocentesis rate of 5\% -that is, all mothers with serum AFP concentrations over the 95 th centile-and $70 \%$ accuracy of gestational dating to \pm 1 week, $32 \%$ of mothers referred for amniocentesis may not actually need it and only $68 \%$ of those who need it will actually have it. The present study was carried out in optimal clinical circumstances and the levels of precision obtained are likely to underestimate rather than overestimate the true extent of the problem.

Clearly, if acceptable results are to be achieved with serum AFP screening, precise gestational dating is required and in a large proportion of cases reliable ultrasound examination will be necessary to supplement clinical findings. This must be taken into account when planning a screening programme for neural tube defects. In some areas it may be more convenient or appropriate for the primary-care physician rather than the hospital clinic to take the blood sample for AFP estimation at the appropriate time, but if he is to be responsible for interpreting results he will require access to ultrasound facilities at least for those of his patients who have uncertain dates or an irregular cycle.

We are grateful to the obstetricians who participated in this study and to the Department of Health and Social Security for giving financial support to the South Wales Anencephaly and Spina Bifida Screening Project, of which this study forms a part.

\section{References}

${ }^{1}$ UK Collaborative Study on Alpha-fetoprotein in Relation to Neural-Tube Defects, Lancet, 1977, 1, 1323.

${ }^{2}$ Chamberlain, R, et al, British Births 1970, vol 1. London, Heinemann, 1970.

${ }^{3}$ Beazley, J M, and Underhill, R A, Nursing Times, 1971, 67, 1414.

4 Dubowitz, L M S, Dubowitz, V, and Goldberg, C, Fournal of Pediatrics, 1970, 77, 1 .

${ }^{5}$ Campbell, S, and Newman, G B, Fournal of Obstetrics and Gynaecology of the British Commonwealth, 1971, 78, 513.

${ }^{6}$ Ferguson Smith, M A, et al, Lancet, 1978, 1, 1330.

${ }^{7}$ Bennett, M J, et al, Lancet, 1978, 2, 1296.

(Accepted 28 February 1979)

\title{
Relation between plasma hormone profiles, symptoms, and response to oestrogen treatment in women approaching the menopause
}

\author{
S CHAKRAVARTI, W P COLLINS, M H THOM, J W W STUDD
}

British Medical fournal, 1979, 1, 983-985

\section{Summary and conclusions}

Out of a consecutive series of 300 patients seen at a menopause clinic, 82 complained of symptoms generally associated with the climacteric, although they were still menstruating. Vasomotor disturbances were absent in 42 of these patients (group 1) and present in 40 (group 2). Headaches, insomnia, and dyspareunia were the most common complaints among the women with vasomotor symptoms, whereas loss of libido and depression predominated in those without. Conjugated equine oestrogens (Premarin) $1.25 \mathrm{mg}$ daily given for three weeks out of four relieved nearly all symptoms in group 2 , but in group 1 the response was disappointing.

The mean plasma oestradiol concentration in women with vasomotor symptoms was significantly lower than that observed during days 1-10 of the menstrual cycle, but plasma testosterone values were not significantly different from those observed in younger women. Plasma follicle-stimulating hormone (FSH) and luteinising hormone (LH) concentrations were similar to those seen

King's College Hospital, London SE5 9RS

S CHAKRAVARTI, FRCS, MRCOG, senior registrar

W P COLLINS, DSC, reader in clinical endocrinology

M H THOM, MRCOG, research senior registrar

J W W STUDD, MD, MRCOG, consultant obstetrician and gynaecologist after the menopause. Concentrations of these hormones in the women without vasomotor symptoms were similar to those in the younger, regularly menstruating women. After six months of oestrogen treatment patients in group 2 had a 2.1-fold increase in mean plasma oestradiol concentration, and plasma FSH and LH concentrations were reduced to $39 \%$ and $66 \%$ of their pretreatment values respectively; in group 1 , however, no such pronounced changes occurred.

High concentrations of FSH were present in patients with oestrogen-responsive symptoms, $15 \mathrm{U} / 1$ being the diagnostic cut-off point. This measurement in the presence of characteristic symptoms therefore constitutes the best method of selecting patients for oestrogen-replacement therapy.

\section{Introduction}

The transition from reproductive life to the postmenopausal years is often protracted and commonly associated with oestrogen-deficiency symptoms which may be confused with commonplace life stresses. Symptoms due to oestrogen deficiency in premenopausal women are easily overlooked, ${ }^{1}$ and Jaszmann, ${ }^{2}$ in an epidemiological study, found that vasomotor symptoms and depression and irritability not only could precede the disappearance of periods by several years but were often at their most severe before the menopause. An essential feature of any treatment is accurate diagnosis, since otherwise the indications for oestrogen replacement may be extended incorrectly to patients with domestic and personality problems. We have therefore sought a means to distinguish true symptoms of 